\title{
Revisión Bibliográfica: Estrategia del Aprendizaje basado en Fenómenos
}

\author{
Vilchis Estrada Alejandra ${ }^{1}$ \\ fbagens@hotmail.com \\ https://orcid.org/0000-0003-0307-0453 \\ Ruíz Espinoza Francisco Higinio ${ }^{1}$ \\ fruiz@uabcs.mx \\ https://orcid.org/0000-0002-0815-3007 \\ Estrada Cervantes René ${ }^{2}$ \\ rene_estrada_cervantes@yahoo.com.mx \\ https://orcid.org/0000-0003-1151-195X \\ Estudiantes de Doctorado en Educación \\ Instituto de Estudios Superiores en Educación por Competencias ${ }^{1}$ \\ Asesor de trabajo doctoral $^{2}$
}

\section{RESUMEN}

El Aprendizaje Basado en Fenómenos al centrarse en la interdisciplinariedad, y puede ser la base para el desarrollo de habilidades de comunicación, pensamiento crítico, creatividad, colaboración y visión global del contexto en los estudiantes desde la primera etapa escolar. Por lo que, al ser una combinación de creencias y prácticas del educando, ésta no incluye reglas y se rige por la investigación activa del alumno o de los alumnos. $\mathrm{Su}$ estrategia es que el estudiante se enfrente a la realidad diaria en el aula, ya sea, con proyectos o una investigación que envuelva su realidad. Hoy en día, la escuela es un escenario en el que conviven, se practican diversas teorías y métodos de enseñanzaaprendizaje, las cuales deben ser mediadas por el profesor-facilitador, ya que la educación no puede ser una reproducción mecánica de conceptos, sino, una oportunidad para generar pensamiento crítico y reflexivo. Por lo que en ABF el estudiante deja de ser pasivo y su rol es activo, ya que desde el punto de partida de las clases son las interrogantes, las ideas, fenómenos, entre otros. Dentro de este modelo, los estudiantes día a día se capacitan en los proyectos a tratar, puesto que son ellos quienes se preparan a profundidad para compartir dicho proyecto con sus compañeros de clase.

Palabras clave: creatividad, educación, fenómenos, motivación. 


\title{
Bibliographic Review: Phenomena-based Learning Strategy
}

\begin{abstract}
Phenomena-Based Learning by focusing on interdisciplinarity, and can be the basis for the development of communication skills, critical thinking, creativity, collaboration and global vision of the context in students from the first school stage. Therefore, being a combination of beliefs and practices of the learner, it does not include rules and is governed by the active investigation of the student or students. Its strategy is that the student faces the daily reality in the classroom, either with projects or an investigation that involves their reality. Nowadays, the school is a scenario where different theories and teaching-learning methods coexist and are practiced, which must be mediated by the teacher-facilitator, since education cannot be a mechanical reproduction of concepts, but an opportunity to generate critical and reflective thinking. Therefore, in ABF the student ceases to be passive and his role is active, since the starting point of the classes are the questions, ideas, phenomena, among others. Within this model, students are trained on a daily basis in the projects to be addressed, since they are the ones who prepare themselves in depth to share the project with their classmates.
\end{abstract}

Keywords: creativity, education, phenomena, motivation

Artículo recibido: 15 abril 2021

Aceptado para publicación: 19 abril 2021

Correspondencia: fruiz@uabcs.mx

Conflictos de Interés: Ninguna que declarar 


\section{INTRODUCCIÓN}

El Phenomenon-Based Learning o Aprendizaje Basado en Fenómenos (ABF), es una iniciativa pedagógica que permite enfocar el aprendizaje de una manera multidisciplinaria, la educación escolar debe renovarse para atender de mejor manera las necesidades de un mundo en constante cambio (Guzmán, 2014). En el cual, los profesionales de la educación junto con planes educativos renovados, tienen como propósito brindar a los estudiantes herramientas y habilidades al concluir la educación básica para ponerlas en práctica una vez que se enfrenten a diversos retos personales y profesionales formando un futuro sostenible (LGE, 2019).

El contexto de orientación pedagógica asociado al ABF se fundamenta en principios de construcción de conocimiento (Dewey, 1916) donde los estudiantes según Huber (2008) aprenden desde sus saberes y experiencias.

Silander (2015) y Symeonidis \& Schwarz (2016) consideran que el ABF tiene la base del constructivismo, por lo que incluye elementos del aprendizaje basado en problemas, el aprendizaje progresivo y el aprendizaje sociocultural. En este sentido, Valanne et al (2017) confirma que el ABF incluye activamente a los estudiantes en actividades prácticas en busca de respuestas a preguntas y resolver problemas.

Dentro de este modelo pedagógico, es esencial que el estudiante sea el líder de investigación ya que, de esta manera, es él mismo quien identifica aquellos vacíos que aún tiene en cuanto a su conocimiento (Fernández, 2018). El ABF se enfatiza en el aprendizaje cooperativo y finalmente el profesor es quien modera las conclusiones que grupalmente se han establecido (Bobrowsky et al., 2014).

Por su parte el papel del docente distinto al del formato tradicional de clases, se convierten en asesores y orientadores, por lo que los educandos tienen un rol más activo, ya que ellos son quienees proponen la forma de cómo abordar el tema y mientras los profesores brindan retroalimentación, con base en las ideas que cada alumno desarrolla (Symeonidis y Schwarz, 2016). Asimismo, durante el desarrollo de estos proyectos se eligen diferentes formatos para presentarlos, entre los que se pueden considerar estan: una presentación, un ensayo, un video, animación, o cualquier otro que se considere apropiado, sugerido por el docente o por el mismo alumno (Briceño et al., 2009).

En esta estrategia de aprendizaje basado en fenómenos un lugar preponderante lo ocupa la tecnología, ya que es usada como medio para obtener información y para presentar los 
resultados finales de la investigación realizada por cada persona o equipo (Villalpando, 2020). Si se realizan trabajos en equipo, se busca enfatizar en el respeto por la diferencia y en el aprendizaje interdisciplinar que busca aprovechar las distintas cualidades de cada educando, unos pueden ser mejores con los números, mientras que otros son mejores dibujando o escribiendo; juntos como equipo, obtendrán un mejor resultado.

Por lo que el objetivo de la estrategia es buscar el desarrollo de habilidades importantes para el futuro, como la comunicación, colaboración, creatividad, pensamiento crítico, comprensión de lectura y crear una visión global del entorno desde temprana edad. Sin embargo, en el momento de las evaluaciones estandarizadas convencionales puede que se presenten inconvenientes, sin duda el Aprendizaje Basado en Fenómenos brinda bases esenciales para las nuevas generaciones.

\section{Finlandia y su propuesta de reforma educativa}

Prakash (2019), establece que Finlandia, en la carrera por mejorar, ha vuelto a la base del fundamento de su sistema educativo. La enseñanza multidisciplinaria se consideraba una herramienta para hacer que la educación sea más relevante y prácticamente aplicable antes en plan de estudios básico para preprimaria en la educación básica de 2004 (FNBE, 2004). Durante la última década, junto con la plétora de cambios que el 2004 trajo consigo en el cambio curricular, la educación finlandesa se había alejado de algunas de sus mejores prácticas anteriores, como la autonomía y la flexibilidad entregado a los profesores. Pero con el cambio curricular de 2014, se hizo un intento para que se revivieran algunas de estas prácticas no olvidadas (FNBE, 2016; FNBE, 2014) y uno de los más reportados erróneamente fue el concepto de Aprendizaje basado en fenómenos $(\mathrm{ABP})$, que fue denominado por muchas revistas (Journals) de renombre y periódicos como la "abolición de la enseñanza de materias (Garner, 2015).

El sistema educativo de Finlandia ha sido considerado uno de los mejores a nivel mundial en las últimas dos décadas. La Organización para la Cooperación y Desarrollo Económicos (OCDE) en su Programa de Evaluación Internacional de Alumnos (PISA por sus siglas en inglés) lleva a cabo un estudio a nivel internacional en el que se evalúan diversas competencias como lectura, matemáticas y ciencias cada tres años en estudiantes de 15 años, en esta prueba, Finlandia a estado destacando con un mayor rendimiento (Symeonidis y Schwarz, 2016). 
Finlandia ha sido uno de los países que decidió renovar su plan de estudios básico para preprimaria y bachillerato. Halinen (2014) Jefe de Desarrollo Curricular de la Junta Nacional de Educación en Finlandia, menciona que "El mundo está cambiando alrededor de la escuela" y el impacto desde la globalización en el año 2000, ha generado desafíos cada vez mayores para un futuro sostenible (Walker, 2016).

En un análisis y revaloración del plan de estudios, fue necesario replantear de acuerdo al nuevo contexto que herramientas y habilidades se requieren desde los contenidos para la enseñanza y el aprendizaje. Sin dejar de lado que es necesario seguir fortaleciendo las habilidades de pensamiento, interacción social, habilidades de procesamiento de información y tecnología (Prakash 2019).

La reforma a el plan de estudios tuvo como propósito, ayudar a los educadores a enseñar lo que los estudiantes necesitan desarrollar desde sus habilidades, motivación e intereses para forjar su futuro sostenible una vez que concluyan la educación básica y se enfrenten a la vida real.

Algunos de los beneficios que surgen a partir de esta renovación en su reforma educativa son:

- Introducción de nuevos conocimientos, entornos y materiales digitales para las escuelas

- Reforma de la educación profesional

- Aceleración de la transición al trabajo

- Mejor acceso al arte y la cultura

- Cooperación entre instituciones y empresas de educación superior

- Iniciativas de garantía juvenil

En este sentido Jäppinen (2017) menciona que el liderazgo educativo, en el cambio o la reforma se ha visto cada vez más como un proceso en lugar de un resultado en el contexto de la educación y las organizaciones que trabajan en el campo de la educación.

Sahlberg (2011) identificó 5 razones detrás del sistema educativo finlandés:

- La escuela obligatoria a partir de los 9 años, ofreciendo igualdad educativa para todos sin importar condiciones económicas.

- La enseñanza es una profesión que inspira a muchos jóvenes finlandeses en su aprendizaje.

- Tiene una política inteligente para la rendición de cuentas. 
- Las personas en Finlandia, confían en sus escuelas.

- El sistema educativo finlandés tiene un liderazgo sostenible y estabilidad política.

\section{Características del Aprendizaje Basado en Fenómenos}

El enfoque basado en fenómenos en la enseñanza y el aprendizaje, surge de un trabajo multidisciplinario en el cual se deja de lado la enseñanza tradicional de materias. Una característica de esta metodología, radica en que conceptualiza el aprendizaje como un proceso de acumulación guiado en el cual el estudiante toma parte activa de su propio aprendizaje en un ambiente educativo autorregulador, proponiendo metas y resolviendo de manera individual y en colaboración, para ello los profesores hacen participes a los estudiantes en un módulo de aprendizaje multidisciplinario por un año, mediante un proyecto basado en fenómenos o temas Sahlberg (2015).

Los estudiantes aprenden a través de un enfoque pedagógico que involucra el entorno y la experiencia cercana al estudiante. Se da prioridad a las inquietudes surgidas de la experiencia del mundo real que les rodea y se estudian los fenómenos en su contexto, cruzando los límites entre las asignaturas que demanda el análisis de la realidad (Nudelman, 2017).

Mattila y Silander (2015) mencionan que, para poder crear la escuela del futuro, es necesario desarrollar el pensamiento sobre fenómenos en la enseñanza aprendizaje dentro del contexto de una pedagogía digital. Silander, comenta que los fenómenos del mundo real ayudan a comenzar en el aprendizaje siempre que sean analizados o estudiados de manera completa en el contexto real. Es decir, en un fenómeno debe haber:

- Un objeto de observación auténtico (tema de interés real)

- Un marco sistémico para lo que se va a aprender (modelo sistémico)

- Un marco metafórico de las cosas a aprender (modelo análogo)

- Una base motivadora para adjuntar las cosas a aprender.

El Aprendizaje Basado en Fenómenos (ABF), permite a los estudiantes aprender de situaciones del mundo real (fenómenos) en el que estudian su entorno y se les ayuda a desarrollar las competencias esenciales para abordar con éxito la vida. Dependiendo de cuál sea el enfoque específico implementado en el aula, debido a que pueden ser desde un estudio superficial del fenómeno a una aplicación más avanzada de su aprendizaje.

Otra característica de la enseñanza basada en fenómenos, es que los profesores inician con preguntas sobre algún fenómeno de interés a los alumnos y estos construyen sus 
respuestas, Los maestros son facilitadores del proceso de aprendizaje en el que su experiencia es para transmitir, animar y guiar a los educandos (Silander, 2015).

El Aprendizaje Basado en Fenómenos, se diferencia de muchos otros modelos pedagógicos que buscan resolver problemas o fenómenos de manera individual y que se centran en un profesor con un currículo a cubrir. El modelo de ABF busca desarrollar la creatividad y el interés del estudiante en el que la experiencia personal con un fenómeno siempre sea más interesante y atractivo en el proceso de aprendizaje en lugar de ser solo receptor de información.

Es por ello que las materias como las matemáticas, las ciencias, la literatura o las sociales no tienen un valor específico dentro de esta metodología, porque se busca que el estudiante no se limite a un solo saber, sino que investigue, indague de manera transversa y es en ese proceso donde estudiantes y docentes trabajan juntos, en el cual los estudiantes dejan de ser pasivos y pasan a desarrollar un rol activo en su aprendizaje. La finalidad de esta metodología no es memorizar sino explorar el fenómeno a conocer, indagar e identificar lo que sucede dentro de un mundo real de acuerdo a su contexto.

Como se ha mencionado anteriormente, en el Aprendizaje Basado en Fenómenos (ABF), los profesores son facilitadores y guías del proceso de aprendizaje de los alumnos, realizando interrogantes del fenómeno o tema mediante múltiples perspectivas interdisciplinarias, desarrollando actividades en las que ponen en juego la observación, el uso de estrategias, la investigación dentro de un entorno real y significativo para los estudiantes, involucrándolos en el proceso de su aprendizaje mediante la retroalimentación enfocada con preguntas que propicien la reflexión y respuestas recabadas por los descubrimientos de la investigación.

El Aprendizaje Basado en Fenómenos, se deriva de la reforma del enfoque pedagógico en el que se provoca la enseñanza y el aprendizaje centrado en el alumno y su mundo real, en el que la construcción de la enseñanza - aprendizaje no puede ser totalmente instruido. El enfoque pedagógico de la fenomenología considera la enseñanza aprendizaje esencial, evitando recaer o transferir toda la responsabilidad a los estudiantes de sus resultados de aprendizaje, o creer que la labor del docente se reduce.

Autores como Meyer Drawe (1987) y Friesen (2014) argumentan que ni los profesores, ni los estudiantes por sí solos contribuyen al aprendizaje exitoso, en esa relación se 
produce una transformación al trabajo de ambos para desarrollar la capacidad de respuesta.

La interdisciplinariedad ayuda a comprender la naturaleza de un fenómeno desde diversas perspectivas, por lo cual es importante identificar y reconocer la complejidad de este proceso. Algunas situaciones de aprendizaje no se pueden instruir en su totalidad, lo que orilla a renovar la enseñanza dando paso a las experiencias de los estudiantes para poder continuar aprendiendo en un mundo cambiante. Biesta (2012a) argumenta que como profesores, se deben realizar juicios concretos de lo que es deseable en el ámbito de la educación y a los medios de interacción.

Biesta (2012b), mencionan que como docentes no se deben perder las competencias educativas ya que estas permiten destacar que se quiere lograr para la planificación y su implementación.

\section{Factores motivacionales en la enseñanza y el aprendizaje basados en fenómenos}

Según Mattila y Silander (2012), la enseñanza basada en fenómenos motiva a los estudiantes y crea motivación interna, porque:

- El aprendizaje está orientado a las necesidades

- Los estudiantes ven valor útil en las teorías e información en la situación de aprendizaje

- Los propios estudiantes pueden establecer intereses y plantear problemas como puntos de partida para el proceso de aprendizaje

- El aprendizaje comienza con el objetivo de entender los fenómenos del mundo real

- La enseñanza basada en fenómenos se centra en los estudiantes; los alumnos son creadores y actores activos

- Las cosas teóricas a aprender están ancladas en situaciones y fenómenos prácticos

- Métodos, fuentes y herramientas reales se utilizan en la situación de aprendizaje

- El aprendizaje es una actividad consciente y orientada a objetivos; los alumnos conocen las metas de aprendizaje (intencionalidad)

- El aprendizaje se produce en un contexto real y holístico (contextualidad, c.f. individual, tareas pequeñas descontextualizadas y desconectadas como su opuesto)

- El proceso de aprendizaje es un continuo completo orientado a objetivos 


\section{Diseñando propuestas de aprendizaje basado en fenómenos}

Para el diseño de Aprendizaje Basado en Fenómenos, es necesario tomar en cuenta el plan de estudios que se renovó, considerar objetivos, propósitos y planear bajo el enfoque multidisciplinario y de transversalidad clasificando en categorías, subcategorías y objetos de estudio (Tabla 1 y Tabla 2).

Tabla 1. Categorías de análisis

\begin{tabular}{|c|c|c|}
\hline $\begin{array}{c}\text { CATEGORÍA } \\
\text { TEÓRICA GENERAL }\end{array}$ & SUBCATEGORÍAS & INDICADORES \\
\hline \multirow{14}{*}{ PLAN DE ESTUDIOS } & \multirow[b]{3}{*}{ Meta } & Definir el tipo de organización del contenido de estudio. \\
\hline & & Proponer metas realizables y alcanzables. \\
\hline & & $\begin{array}{l}\text { Enunciar los objetivos a alcanzar al final de cada } \\
\text { periodo académico. }\end{array}$ \\
\hline & \multirow{3}{*}{$\begin{array}{l}\text { Contexto del } \\
\text { estudiante }\end{array}$} & $\begin{array}{l}\text { Determinar un sistema de valores con el que se quiere } \\
\text { formar al estudiante. }\end{array}$ \\
\hline & & Comprender las necesidades de los estudiantes. \\
\hline & & Favorecer el proceso de aprendizaje. \\
\hline & \multirow{5}{*}{ Estrategias didácticas } & Promover la participación. \\
\hline & & Evaluar el aprendizaje mediante la retroalimentación. \\
\hline & & $\begin{array}{l}\text { Fomentar la investigación en los } \\
\text { estudiantes. }\end{array}$ \\
\hline & & Establecer los instrumentos didácticos adecuados. \\
\hline & & $\begin{array}{l}\text { Integrar los parámetros del fenómeno a la acción } \\
\text { educativa. }\end{array}$ \\
\hline & \multirow[t]{3}{*}{ Normatividad } & Cumplir una función pedagógica. \\
\hline & & Cumplir una función administrativa. \\
\hline & & Certificar las metas alcanzadas. \\
\hline \multirow{9}{*}{$\begin{array}{l}\text { APRENDIZAJE } \\
\text { BASADO } \\
\text { FENÓMENOS }\end{array}$} & \multirow{3}{*}{$\begin{array}{l}\text { Articulación contexto } \\
\text { aprendizaje }\end{array}$} & $\begin{array}{l}\text { Generar espacios de aprendizaje según } \\
\text { contexto. }\end{array}$ \\
\hline & & Utilizar fenómenos contextuales. \\
\hline & & Promover el interés por el conocimiento de la ciencia. \\
\hline & \multirow{3}{*}{ Papel del docente } & Promover autoconfianza y respeto. \\
\hline & & Generar pensamiento crítico y creativo. \\
\hline & & $\begin{array}{l}\text { Motivar a los estudiantes por medio de la aplicación de } \\
\text { conocimientos. }\end{array}$ \\
\hline & \multirow{3}{*}{$\begin{array}{l}\text { Sentimientos } \\
\text { comportamiento y } \\
\text { atención de los } \\
\text { estudiantes. }\end{array}$} & Favorecer la gestión de las emociones \\
\hline & & Proponer soluciones a los problemas asignados. \\
\hline & & Fomentar un ambiente académico adecuado. \\
\hline
\end{tabular}

Fuente: Medina (2017). 
Tabla 2. Fenomenología de diferentes miradas de la educación en ciencias

\begin{tabular}{|c|c|c|}
\hline & Características & Objeto de estudio y resultados \\
\hline $\begin{array}{l}\text { Fenomenología de } \\
\text { educación científica }\end{array}$ & $\begin{array}{l}\text { - Carácter descriptivo / } \\
\text { analítico. } \\
\text { - Relación estrecha entre } \\
\text { aprendizaje y enseñanza de la } \\
\text { ciencia. } \\
\text { - Relación cotidiana entre } \\
\text { estudiante y maestro. }\end{array}$ & $\begin{array}{l}\text { 1. Profesores y experiencia de los maestros. } \\
\text { - Enfoque central recae en los estudiantes y el } \\
\text { secundario en la tarea de enseñar. } \\
\text { - Frustración y depresión por el trabajo } \\
\text { (docentes). } \\
\text { - Manejo de científico (conceptos - ciencia } \\
\text { instrumental). } \\
\text { 2. Estudiantes como personas. } \\
\text { - La ciencia no inspira a los estudiantes, aun } \\
\text { así, cuando se estudia por horas. } \\
\text { - Mundo científico fragmentado de las } \\
\text { experiencias. } \\
\text { - La educación y la cultura no tienen relación. } \\
\text { 3. Actividades de aprendizaje y enseñanza. } \\
\text { - Resaltar la experiencia en la enseñanza. } \\
\text { - No descubrir individuos aislados, más bien } \\
\text { potencializar generalidades. } \\
\text { Relacionar el mundo científico y la } \\
\text { experiencia cotidiana. }\end{array}$ \\
\hline $\begin{array}{l}\text { Fenomenología en la } \\
\text { educación científica }\end{array}$ & $\begin{array}{l}\text { - Recolección de datos en las } \\
\text { clases de ciencias. } \\
\text { - Sugiere como enseñar } \\
\text { ciencias. } \\
\text { - Enfoque pedagógico de la } \\
\text { fenomenología en fenómenos } \\
\text { de la naturaleza. }\end{array}$ & $\begin{array}{l}\text { - Descripción, reflexión y discusión de los } \\
\text { fenómenos percibidos. } \\
\text { - Identificar las variaciones del mismo } \\
\text { fenómeno o de otros diferentes. } \\
\text { - Utilizar los sentidos para la interpretación } \\
\text { del fenómeno. }\end{array}$ \\
\hline \multirow{2}{*}{$\begin{array}{l}\begin{array}{l}\text { Fenomenología } \\
\text { educación }\end{array} \\
\text { integradas. }\end{array}$} & \multirow{2}{*}{$\begin{array}{l}\text { - Enfatiza en el proceso de } \\
\text { enseñanza y el aprendizaje de } \\
\text { las ciencias. } \\
\text { - Reflexiona sobre los } \\
\text { fenómenos naturales en la } \\
\text { ciencia. } \\
\text { - Estudios de Michael Faraday y } \\
\text { Martin Wagenschein }\end{array}$} & $\begin{array}{l}\text { Michael Faraday } \\
\text { - Ciencia experimental. } \\
\text { - Instrumentación de la ciencia. } \\
\text { - Enseñanza de la ciencia basada en } \\
\text { fenómenos naturales. }\end{array}$ \\
\hline & & $\begin{array}{l}\text { Martin Wagenschein } \\
\text { - Descripción el mundo con modelos } \\
\text { abstractos. } \\
\text { - La ciencia no son solo ilustraciones } \\
\text { experimentales. } \\
\text { - Fenómenos como punto de partida al } \\
\text { acercamiento hacia el mundo científico. } \\
\end{array}$ \\
\hline $\begin{array}{lll}\text { Potencialidades de } & \text { la } \\
\text { fenomenología en } & \text { la } \\
\text { educación científica } & \end{array}$ & $\begin{array}{l}\text { - Comprensión de la naturaleza. } \\
\text { - Actividades de aprendizaje, } \\
\text { enseñanza y autorreflexión. } \\
\text { - Formación del profesorado en } \\
\text { educación científica. }\end{array}$ & $\begin{array}{l}\text { - Desinterés por parte de los estudiantes por } \\
\text { parte de las asignaturas que tienen una } \\
\text { relación directa con la ciencia. } \\
\text { - La fenomenología debe cerrar la brecha entre } \\
\text { el conocimiento científico y el mundo de la } \\
\text { vida. }\end{array}$ \\
\hline
\end{tabular}

Fuente: Camacho y Camargo (2019).

Es necesario contar con un instrumento que permita recolectar la información obtenida en la investigación del fenómeno, con el fin de identificar las relaciones entre conceptos y fenómenos revisados, identificando la pertinencia de los instrumentos didácticos utilizados y los tipos de evaluación, realizar una matriz para identificar y analizar cada categoría del diseño del plan de estudios basado en fenómenos y poderlos mejorar en cada 
versión ya que la matriz es un instrumento de análisis y no de decisión en el cual se analiza.

1. Claridad en la orientación pedagógica.

2. Evidencia del enfoque experimental del plan de estudios.

3. Brevedad y pertinencia

4. Concordancia (conceptual -metodológica)

5. Consideración de las políticas públicas en la construcción del plan de estudios.

6. Pertinencia en la evaluación

7. Claridad y cohesión

8. Factores motivacionales que los estudiantes desarrollaran en cada actividad usando el aprendizaje basado en fenómenos.

9. Evaluación de los procesos enseñanza aprendizaje.

10. Pertinencia y aplicabilidad del plan de estudios.

De esta forma se podrán ver los resultados una vez que se lleva a cabo la aplicación de una propuesta metodológica basada en el Aprendizaje Basado en Fenómenos.

\section{Experiencias del Aprendizaje Basado en Fenómenos}

Østergaard et al (2010), en un estudio en agroecología en que aplican la estrategia del aprendizaje basado en fenómenos reportan que esta metodología proporciona relevancia práctica para que los estudiantes basen sus conocimientos en fincas y comunidades y esta relevancia conduce a una acción responsable de la educación. El tratar con problemas complejos de sostenibilidad requiere reunir una amplia gama de conocimientos especializados en un modo de colaboración interprofesional.

Por su parte Camacho y Camargo (2019) reportan que el aprendizaje basado en fenómenos implementados en la enseñanza de las ciencias naturales, permiten al estudiante empoderarse de los procesos constructivos de aprendizaje para formar explicaciones del mundo de la vida en conexión con las ciencias naturales.

En este mismo sentido Pantoja y Covarrubias (2013) en un trabajo realizado en la enseñanza de la biología en el bachillerato a partir del aprendizaje basado en problemas $(\mathrm{ABP})$ reportan a esta metodología como una alternativa de enseñanza para abordar los temas de biología en la educación media, dejando de lado los métodos tradicionales, esto permitió ampliar los horizontes de conceptualización respecto de los argumentos teóricos para sustentar la metodología ABP. 
Según Francis et al (2013), el aprendizaje de la agroecología basado en los fenómenos proporciona una lógica y una plataforma para crear puentes entre la academia y la sociedad. El aprendizaje basado en experiencias reflexivas en granjas y en comunidades ha proporcionado el fundamento y el núcleo de un curso de agroecología en Noruega desde el año 2000 en la Universidad Noruega de las Ciencias de la Vida (NMBU). Los equipos de estudiantes trabajan con profesores de la Universidad y con las personas interesadas mediante "casos abiertos" con el fin de identificar las principales limitaciones y las posibilidades futuras. Esta estrategia de aprendizaje utiliza situaciones del mundo real en la granja y en la comunidad, donde ni el instructor ni los clientes conocen de entrada las soluciones. Utilizando métodos de las ciencias sociales y de las ciencias naturales, los equipos examinan y evalúan las dimensiones productivas, económicas, ambientales y sociales, integradas en sistemas completos. Después, los estudiantes diseñan y evalúan escenarios futuros y piensan planes de acción. El resultado ha sido una base sólida para la acción responsable en los proyectos futuros de los estudiantes en los campos de la educación y el desarrollo.

Por su parte Briseño et al (2009), establecen que el estudio de ciencias naturales siempre ha representado una gran dificultad para los estudiantes, el apelativo de las tres marías con el que popularmente han sido denominadas (la física, la química y la matemática) y que las ha hecho particularmente conocidas, es indicativo entre otras cosas de lo expresado. Basados en la situación descrita, esta investigación propone como iniciativa de solución la exploración de una herramienta alternativa en formato electrónico para la interpretación de los fenómenos electromagnéticos, teniendo como ejes las nuevas corrientes educativas, considerando los nuevos modelos y herramientas surgidos para la enseñanza, así como también, los avances tecnológicos, la educación asistida por computadora y el desarrollo de páginas Web, en un todo de acuerdo con la realidad del entorno local, para mejorar el proceso de enseñanza-aprendizaje.

Mediante el diálogo interactivo entre el estudiante y la herramienta se logró que el educando sea un participante activo en su proceso de aprendizaje, en vez de un espectador pasivo y que pueda ajustar su proceso de aprendizaje de acuerdo a sus necesidades y disposición de tiempo. Además, los Ítems presentados a expertos, profesores, estudiantes y otros profesionales han ratificado que la herramienta propuesta cumple con los objetivos previstos. 
En un estudio realizado por Valanne et al (2017) donde implementaron el ABP en una escuela de Abu Dabi para mejorar el proceso de Enseñanza-Aprendizaje, en que pretendía aumentar la motivación por la lectura de los jóvenes estudiantes, mejorar sus habilidades lectoras, profundizar y revisar transversalmente el currículo. El método clave para impartir el plan de estudios con este enfoque holístico e interdisciplinario se construyó sobre cuentos e historias. El beneficio general obtenido fue lograr una mayor motivación de los estudiantes por la lectura, en la satisfacción de los padres y en el entusiasmo de colaboración de los maestros para crear un aprendizaje significativo con ABP.

\section{CONSIDERACIONES FINALES}

El enfoque de la enseñanza y el aprendizaje basado en fenómenos puede ser una estrategia que nos permita a abrir los límites de la enseñanza tradicional y avanzar hacia la interdisciplinariedad mediante las exploraciones de fenómenos. Poder entender la naturaleza de un fenómeno desde múltiples perspectivas. Por tanto, es fundamental reconocer y comprender la complejidad del proceso, que bien, no es una actividad fácil, pero tampoco imposible. Esta complejidad también se aplica a situaciones de aprendizaje en la escuela que no se pueden instruir completamente, aunque esto, no hace obsoleta la preparación y la planificación. En un aprendizaje basado en fenómenos es necesario aceptar el hecho de que, ganar una nueva perspectiva requiere dejar atrás los procesos anteriores (viciados o caducos). De esta manera los educadores o facilitadores deben estar preparados para guiar a los estudiantes con superlativo cuidado en este proceso.

Por otra parte al tener una estrecha conexión entre el constructivismo y el enfoque basado en fenómenos nos revela implicaciones específicas para la enseñanza y el aprendizaje. Por lo que, los profesores podría parecer que estan exentos de su responsabilidad de enseñar, debidoa que los significados de los fenómenos surgen en la mente de los estudiantes. En una fenomenología vista cual, pero, los profesores deben ceder el paso a las experiencias de los estudiantes y reconocer los momentos de aprender cuando surgen. Pero también deben asumir compromiso, responsabilidad, trabajo y competencia en el proceso educativo. 


\section{LISTA DE REFERENCIAS}

Biesta, G. 2012a. The future of teacher education: Evidence, competence or wisdom? Research on Steiner Education, 3(1),8-21.

Biesta, G. 2012b. Giving teaching back to education: responding to the disappearance of the teacher. Phenomenology \& Practices, 6(2),35-49.

Bobrowsky, M, Korhonen., M, \& Kohtamaki. (2014). Using Physics Gadgets and Gizmos, Grades 9-12: Phenomenon-Based Learning. Recuperado de https://www.nsta.org/store/product_detail.aspx?id=10.2505/9781936959365.

Briceño, Jesús, Rosario, Jesús, Rivas, Yasmelis, Lobo, Hebert, Gutiérrez, Gladys, Villarreal, Manuel, Díaz, Juan, \& Pineda, Francisco. (2009). El aprendizaje de fenómenos electromagnéticos mediante una herramienta interactiva. Educere, 13(45), 501-507.

Camacho, R. D. y Camargo, Y. D. 2019. El fenómeno de la reforestación y el aprendizaje basado en fenomenos como perspectiva de enseñanza en las ciencias naturales. Tesis de

Maestría.

https://repository.udistrital.edu.co/bitstream/handle/11349/22878/Camacho\%20Ro mero\%20Diego\%20Francisco\%202019.pdf? sequence=1\&isAllowed=y

Dewey, J. (1916). Democracy and Education: An Introduction to the Philosophy of Education. American Journal of Sociology, 22(5), 674-676. Doi:10.2307 / 2178611.

Fernández, M. 2018. Más escuela y menos aula. Madrid: Morata.

Finnish National Board of Education [FNBE]. (2014). National core curriculum for basic education 2014. Retrieved from https://www.oph.fi/english/curricula_and_qualifications/basic_educat ion/curricula_2014.

Finnish National Board of Education [FNBE]. (2016). National core curriculum for general upper secondary schools 2015 . Retrieved from.

Francis, C., Breland, T. A., Østergaard, E., Lieblein, G., \& Morse, S. (2013). Aprendizaje de la agroecología basado en los fenómenos: Un prerrequisito para la transdisciplinariedad y la acción responsable. Agroecología, 8(2), 45-54. Doi: https://doi.org/10.6018/agroecologia.

Friesen, N. 2014. Waldenfels' Responsive Phenomenology of the Alien: An Introduction. Phenomenology and Practice,7(2), 68-77. 
Garner R. 2015. Finland schools: Subjects scrapped and replaced with 'topics' as country reforms its education system. Retrieved from https://www.independent.co.uk/news/world/europe/finland-schoolssubjects-are-outand-topics-are-in-as-country-reforms-its-educationsystem-10123911.html

Guzmán, C. (2014). Finlandia: viaje por uno de los mejores sistemas educativos del mundo, Periódico El Tiempo. Recuperado de: http://www.eltiempo.com/archivo/documento/CMS-14678295.

Huber, G. 2008. Aprendizaje activo y metodologías educativas. Revista de Educación, número extraordinario (1):59-81. http://www.educacionyfp.gob.es/dam/jcr:14edd70f-c97a-4361-8757ef0c83ce5bea/re200804-pdf.pdf.

LGE [Ley General de Educación] (2019). Diario Oficial de la Federación. México. Recuperado de: https://dof.gob.mx/nota_detalle.php?codigo=5573858\&fecha=30/09/2019.

Mattila, P. \& Silander, P. 2015. How to create the school of the future: Revolutionary thinking and design from Finland. Oulu: University of Oulu, Center for Internet Excellence. School Innovation and Learning Center (SILC) - project funded by European Social Fund (ESF). https://www.classter.com/wpcontent/uploads/2016/09/How-to-create-the-school-of-the-future.pdf.

Medina, G. D. 2017. Conceptos y fenómenos: propuesta de reforma del plan de estudios $\begin{array}{llll}\text { en } & \text { el } & \text { bachillerato, }\end{array}$ http://dx.doi.org/10.14483/udistrital.jour.RC.2017.27.a2

Meyer-Drawe, K. (1987a). Der phänomenologische Blick: über die Schwierigkeiten, die noch stumme Erfahrung zur Aussprache ihres eigenen Sinnes zu bringen. In M. Hellemans \& P. Smeyers (Eds.), Phänomenologische Pädagogik: Methodologische und theoretische Aufsätze: Tagungsbericht des 3. Arbeitskreises für phänomenologisch-pädagogische Forschungen in Leuven 1985, 13-24.

Østergaard, E., Lieblein G., Breland, T.A., Francis C. 2010. Students Learning Agroecology: Phenomenon-Based Education for Responsible Action. Journal of Agricultural Education and Extension 16(1), 23-37. Doi: $\underline{10.1080 \quad /}$ $\underline{13892240903533053}$ 
Prakash, N. R. 2019. Phenomenon-Based Learning in Finland. Master's Thesis in Education Spring Term. Department of Education University of Jyväskylä. https://jyx.jyu.fi/bitstream/handle/123456789/64611/1/URN\%3ANBN\%3Afi\%3Aj yu-201906143197.pdf

Prime Minister's Office Finland. 2015. Finland, a land of solutions. Strategic Programme of Prime Minister Juha Sipilä's Government. Retrieved October 5, 2016, from http://vnk.fi/en/publication?pubid=6407.

Pantoja Castro, Julio César, \& Covarrubias Papahiu, Patricia. (2013). La enseñanza de la biología en el bachillerato a partir del aprendizaje basado en problemas (ABP). Perfiles educativos, 35(139), 93-109.

Silander, P. (2015). The school of the future. Published by Helsinki City Education Department. $\quad$ http://www.phenomenaleducation.info/phenomenon-basedlearning.html.

Symeonidis, V., Schwarz J.F. 2016. Phenomenon-Based Teaching and Learning through the Pedagogical Lenses of Phenomenology: The Recent Curriculum Reform in Finland. Forum Oświatowe, 28(2), 31-47.

Valanne, E. A., Al Dhaheri, R. M., Kylmalahti, R., Sandholm, R. H. 2017. Phenomenon Based Learning Implemented in Abu Dhabi School Model. International Journal of Humanities and Social Sciences, 9(3),1-17. https://ijhss.net/index.php/ijhss/article/view/263/106.

Villalpando, I. 2020. La escuela mexicana ante la pandemia: diagnóstico y escenarios posibles. Faro Educativo, Apunte de Política, (9),3-7. 